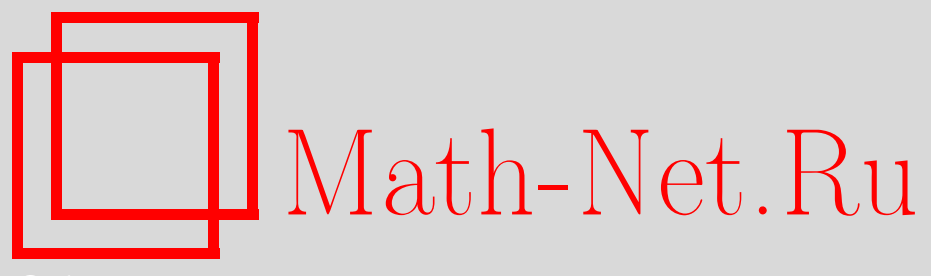

Р. В. Шамин, Аппроксимация эволюционных дифференциальных уравнений в шкалах гильбертовых пространств, Матем. заметки, 2009, том 85, выпуск 2, 318 320

DOI: https://doi.org/10.4213/mzm5259

Использование Общероссийского математического портала Math-Net.Ru подразумевает, что вы прочитали и согласны с пользовательским соглашением http://www . mathnet.ru/rus/agreement

Параметры загрузки:

IP: 54.84 .234 .179

26 апреля 2023 г., 18:23:33

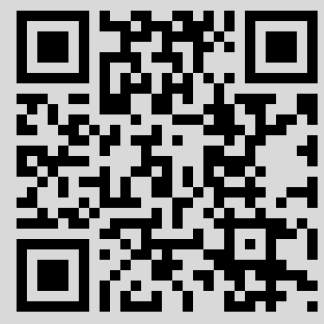




\section{Аппроксимация эволюционных дифференциальных уравнений в шкалах гильбертовых пространств}

\section{Р. В. Шамин}

K эволюционным дифференциальным уравнениям в гильбертовых пространствах относится большое количество задач, возникающих в различных областях естествознания. При этом возникает необходимость в аппроксимации бесконечномерных систем конечномерными уравнениями (системами обыкновенных дифференциальных уравнений). При аппроксимации эволюционных уравнений в шкалах гильбертовых пространств возможно достичь высокой эффективности при использовании вычислительной техники. В настоящей работе рассматривается вопрос об оценке времени существования решений и аппроксимаций в выбранных шкалах пространств. Приведенные результаты позволяют строить конструктивные методы для получения такой оценки в конкретных случаях. Заметим, что вопрос о времени существования решения в шкалах пространств актуален как для линейных уравнений, для которых имеют место результаты о глобальной разрешимости, так и для нелинейных систем, для которых время существования решений конечно.

Пусть $H$ - гильбертово пространство. Всюду в статье гильбертовы пространства предполагаются сепарабельными и бесконечномерными. Через $e_{k}$ обозначим ортонормированный базис в $H$. Введем непрерывный, вообще говоря, нелинейный оператор $A: D \rightarrow H$, где $D$ - гильбертово пространство, плотно и непрерывно вложенное в $H$, такое, что $\left\{e_{k}\right\} \subset D$. Будем рассматривать задачу Коши

$$
\begin{gathered}
u^{\prime}(t)=A u(t), \quad t \in(0, T) \\
u(0)=\varphi, \quad \varphi \in H .
\end{gathered}
$$

ОПРеДЕЛЕниЕ 1. Решением задачи $(1),(2)$ на $(0, T)$ будем называть функцию $u \in$ $L_{2}(0, T ; D), u^{\prime} \in L_{2}(0, T ; H)$, удовлетворяющую $(1),(2)$.

ЗАмечание 1 . В силу замечания 2.2 [1] функция $u \in L_{2}(0, T ; D), u^{\prime} \in L_{2}(0, T ; H)$ имеет след $u(0) \in H$.

Для построения шкалы гильбертовых пространств рассмотрим определенную для $k=$ $1,2, \ldots$ и $s \geqslant 0$ систему функций $\left\{\gamma_{k}(s)\right\}$ такую, что для каждого фиксированного $k$ имеет место: $\gamma_{k}(s)>0, \gamma_{k}(0)=1, \gamma_{k}(s)$ непрерывны и строго убывают по $s$. Пространства $H_{s}$, $s \geqslant 0$, введем как замыкание всех линейных оболочек $\sum_{k=1}^{N} u_{k} e_{k}$ по норме

$$
\|u\|_{H_{s}}^{2}=\sum_{k=1}^{\infty}\left|u_{k}\right|^{2} \gamma_{k}^{-2}(s), \quad \text { где } \quad u_{k}=\left(u, e_{k}\right)_{H} .
$$

Введем конечномерный проектор $P_{N}$ в пространстве $H$ по формуле

$$
P_{N}\left(\sum_{k=1}^{\infty} u_{k} e_{k}\right)=\sum_{k=1}^{N} u_{k} e_{k}
$$

Задачу (1), (2) будем аппроксимировать конечномерными задачами

$$
\begin{gathered}
\left(u^{N}\right)^{\prime}(t)=P_{N} A u^{N}(t), \quad t \in(0, T) \\
u^{N}(0)=P_{N} \varphi, \quad \varphi \in H .
\end{gathered}
$$

Работа выполнена при поддержке Российского фонда фундаментальных исследований (гранты №№ 07-01-00268-а, 07-05-00648-a, 07-05-92211-НЦНИЛ_а). 
ПрЕДПОЛОЖЕНИЕ 1. СУществуют такие числа $s_{l}>0 u t_{l}>0, l=0,1, \ldots$, что при всех $N$ задача (3),(4) имеет единственное решение $u^{N}(t) \in H_{s_{l}}$ при $t \in\left(0, t_{N}\right)$, а задача (1),(2) имеет единственное решение и на $\left(0, t_{0}\right)$, и при почти всех $t \in\left(0, t_{0}\right)$ это решение принадлежит $H_{s_{0}}$. При этом для некоторого $T>0$ имеет место

$$
\lim _{N \rightarrow \infty}\left\|u^{N}-u\right\|_{L_{2}\left(0, T ; H_{s_{0}}\right)}=0 .
$$

Для любого элемента $u \in H_{s}, s>0$, введем функцию

$$
\nu(u)=\varliminf_{k \rightarrow \infty}=\nu_{k}\left(u_{k}\right),
$$

где функция $\nu_{k}\left(u_{k}\right)$ такая, что $\gamma_{k}\left(\nu_{k}\left(u_{k}\right)\right)=\left|u_{k}\right|$ для $1 \geqslant\left|u_{k}\right|>0$, для $u_{k}>1$ полагаем $\nu\left(u_{k}\right)=0$, для $u_{k}=0$ имеем $\nu(0)=\infty$.

Teоpema 1. Пусть $u \in H_{s}$, mогда $\nu(u) \geqslant s$.

ДоказАтельство. Имеем представление $\left|u_{k}\right|=\alpha_{k} \gamma_{k}(s)$, где $\alpha_{k} \in l_{2}$. Без ограничения общности будем считать, что $\alpha_{k}<1$. Поскольку функция $\nu_{k}(a)$ не возрастает, имеем

$$
\nu_{k}\left(\left|u_{k}\right|\right)=\nu_{k}\left(\alpha_{k} \gamma_{k}(s)\right) \geqslant \nu_{k}\left(\gamma_{k}(s)\right)=s .
$$

Теорема 2. Предположим, что ряд

$$
\sum_{k=1}^{\infty}\left(\frac{\gamma_{k}\left(s_{2}\right)}{\gamma_{k}\left(s_{1}\right)}\right)^{2}
$$

сходится для $0<s_{1}<s_{2}$, тогда для $u \in H_{0}$ такого, что $\nu(u)=s, 0<s<\infty$, имеет место

$$
u \in H_{s-\varepsilon} \quad \text { для любого } \varepsilon \in(0, s) .
$$

ДоказАтельство. Пусть фиксировано $\varepsilon \in(0, s)$. Обозначим $\delta_{k}=\nu_{k}\left(u_{k}\right)-s$. Поскольку $\underline{\lim }_{k \rightarrow \infty} \nu_{k}\left(u_{k}\right)=\nu(u)=s$, существует такое $K>0$, что $\delta_{k}>-\varepsilon$ при $k \geqslant K$. В этом случае имеем $\left|u_{k}\right|=\gamma_{k}\left(s+\delta_{k}\right), k \geqslant K$. В силу условия на $\gamma_{k}(s)$ получаем

$$
\|u\|_{H_{s-\varepsilon}}^{2}=\sum_{k=1}^{K-1}\left|u_{k}\right|^{2} \gamma_{k}^{-2}(s-\varepsilon)+\sum_{k=K}^{\infty}\left(\frac{\gamma_{k}\left(s_{2}\right)}{\gamma_{k}\left(s_{1}\right)}\right)^{2}<\infty .
$$

Для важного в приложениях случая, когда $\gamma_{k}(s)=e^{-k s}$, получен результат об оценке функции $\nu(u)$ по значениям $\nu_{k}\left(u_{k}^{N}\right)$.

Теорема 3. Пусть $\gamma_{k}(s)=e^{-k s} ;$ предположим, что выполнено предположение $1 u$ функции $u^{N}$ суть решения задачи (3),(4), а функция и есть решение задачи (1),(2). Для $t \in(0, T)$, где $T$ - величина из предположения 1 , если $\nu(u(t))=s, 0<s<\infty$, то для любого $0<\varepsilon<s$ существует константа $C_{\varepsilon}>0$, не зависящая от $N u k$, такая, что

$$
\nu_{k}\left(u_{k}^{N}(t)\right)-\nu(u(t)) \leqslant C_{\varepsilon} \frac{1}{k}+\varepsilon, \quad k \leqslant N .
$$

Доказательство теоремы 3 обобщает доказательство теоремы 2.1 [2].

В качестве примера рассмотрим абстрактные параболические уравнения. Пусть $V-$ гильбертово пространство, плотно и непрерывно вложенное в $H$. Рассмотрим линейный непрерывный оператор $L: V \rightarrow V^{\prime}$, являющийся $V$-коэрцитивным, т.е. $\operatorname{Re}\langle L v, v\rangle \geqslant\|v\|_{V}^{2}$ для любого $v \in V$. Введем замкнутый неограниченный оператор $L: D(L) \subset H \rightarrow H$, где

$$
D(L)=\{u \in V: L u \in H\} .
$$


Оператор в уравнении (1) зададим следующим образом:

$$
A u(t)=-L u(t)+f(t), \quad \text { где } \quad f \in L_{2}(0, T ; H) .
$$

В работах [1], [3] приведены примеры $V$-коэрцитивных операторов из числа функционально-дифференциальных операторов, которые имеют важные приложения (см. [4]). Используя результаты упомянутых работ, можно показать, что при $\varphi \in V$ выполнено предположение 1.

Другим важным примером являются нелинейные системы Коши-Ковалевской. Такие системы в гильбертовых пространствах изучались во многих работах, например, [5]-[8]. В работе [2] теорема 3 применялась для получения конструктивных оценок времени существования аналитических решений для нелинейных систем Коши-Ковалевской. В работе [9] эти результаты позволили решить важный вопрос о разрешимости уравнений, описывающих поверхностные волны идеальной жидкости, на конечном интервале по времени.

Автор выражает глубокую благодарность проф. А.Л. Скубачевскому за внимание к работе и ряд важных советов.

\section{СПИСОК ЦИТИРОВАННОЙ ЛИТЕРАТУРЫ}

[1] Р. В. Шамин, Матем. сб., 194:9 (2003), 141-156. [2] Р. В. Шамин, Труды семинара по дифференциальным и функиионально-дифференциальным уравнениям в РУДН под руководством А.Л. Скубачевского, СМФН, 21, РУДН, М., 2007, 133-148. [3] Р. В. Шамин, Матем. заметки, 71:4 (2002), 636-640. [4] A. L. Skubachevskii, Elliptic Functional-Differential Equations and Applications, Oper. Theory Adv. Appl., 91, Birkhäuser Verlag, Basel, 1997. [5] Л.В. Овсянников, Докл. АН СССР, 200:4 (1971), 789-792. [6] T. Nishida, J. Differential Geom., 12:4 (1977), 629-633. [7] F. Tréves, Ovcyannikov Theorem and Hyperdifferential Operators, Notas Mat., 46, Instituto de Mathemática Pure e Aplicada, Rio de Janeiro, 1968. [8] Ю.А. Дубинский, Задача Коши в комплексной области, Изд-во МЭИ, М., 1996. [9] Р. В. Шамин, Вычислителъные эксперименты в моделировании поверхностных волн в океане, Наука, М., 2008.

\section{Р. В. Шамин}

Поступило

Институт океанологии им. П. П. Ширшова РАН, Москва

10.07.2008

E-mail: roman@shamin.ru 